\title{
Seroprevalencia de Bordetella pertussis en el personal de enfermería y médicos residentes del Hospital Infantil de México Federico Gómez
}

\author{
Esperanza Navarrete, Almudena Laris-González, Arturo D. Castro-Díaz, Gustavo Rosales-Pedraza, \\ Sarbelio Moreno-Espinosa y Daniela de la Rosa-Zamboni* \\ Hospital Infantil de México Federico Gómez, Ciudad de México, México
}

\begin{abstract}
Resumen
Introducción: Bordetella pertussis es el agente causal de la tosferina, una enfermedad de alta letalidad, especialmente en menores de 6 meses, pero prevenible mediante la vacunación. Los reportes en hospitales de brotes de tosferina muestran que el caso índice suelen ser personas adultas. En adultos, la enfermedad se manifiesta principalmente con tos persistente. El propósito de este estudio fue conocer la seroprevalencia de B. pertussis en el personal de salud de un hospital pediátrico en un país donde aún no se considera la vacunación obligatoria para los empleados. Métodos: Participaron personal de enfermería y médicos residentes en trato directo con pacientes hospitalizados. A cada participante se le realizó detección de anticuerpos inmunoglobulina G, antitoxina de pertussis (anti-TP) y se le aplicó un cuestionario para datos clínicos y demográficos. Resultados: Se incluyeron 93 individuos, el 85\% de personal de enfermería con mediana de edad de 35 años (rango intercuartil: 29-42.5). El 21.5\% de los participantes laboraban en el Servicio de Urgencias, el 8.6\%, en la Unidad de Terapia Intensiva Pediátrica, el 6.5\%, en la Unidad de Cuidados Intensivos Neonatales. Se encontraron títulos detectables de anticuerpos anti-TP en el 18.3\%, de los cuales, el 53\% presentaron títulos de infección reciente y solamente el 23.5\%, historia de tos de más de dos semanas de evolución. Conclusiones: El personal de salud está en riesgo de sufrir la enfermedad y de transmitirla a los lactantes, quienes pueden fallecer por esta causa. Este estudio sugiere que las políticas actuales de vacunación en personal de salud se deben de modificar para determinar obligatoriedad de la vacuna, especialmente en quienes atienden a la población pediátrica.
\end{abstract}

Palabras clave: Seroprevalencia. Bordetella pertussis. Profesional de la salud.

\section{Seroprevalence of Bordetella pertussis in pediatric healthcare workers at the Hospital Infantil de México Federico Gómez}

\begin{abstract}
Background: Bordetella pertussis is the causative agent of pertussis, a disease that is preventable by vaccination but has a high mortality, particularly in children $<6$ months. Reports of pertussis outbreaks in hospitals show that the index case is usually an adult. In adults, the disease manifests mainly with persistent cough. The purpose of this study was to determine
\end{abstract}

*Daniela de la Rosa-Zamboni E-mail: rzdaniela@ hotmail.com
Disponible en internet: 13-05-2019 Bol Med Hosp Infant Mex. 2019;76:120-125 www.bmhim.com 
the seroprevalence of $B$. pertussis in the health personnel of a pediatric hospital in a country where vaccination of this staff is not considered mandatory. Methods: Nursing staff and resident doctors who were involved in direct treatment with hospitalized patients participated in the study. Each participant was screened for immunoglobulin $G$ anti-pertussis toxin antibodies (anti-PT), and a questionnaire was applied for clinical and demographic data. Results: Ninety-three individuals were included, of which $85 \%$ were nurses, median age 35 years (interquartile range: 29-42.5). The participants worked in the emergency department (21.5\%), in the Pediatric Intensive Care Unit (8.6\%), and in the Neonatal Intensive Care Unit (6.5\%). Detectable titers of anti-TP antibodies were found in $18.3 \%$, of which $53 \%$ presented titles suggestive of recent infection and only $23.5 \%$ cough $>2$ weeks of duration. Conclusions: Health personnel are at risk of suffering from the disease and be potential transmitters to infants, who may die from this cause. This study suggests that the current vaccination policies in health personnel should be modified to determine the compulsory nature of the vaccination, especially in those individuals in charge of the care of the pediatric population.

Key words: Seroprevalence. Bordetella pertussis. Healthcare professional.

\section{Introducción}

Bordetella pertussis es el agente causal de la tosferina, una enfermedad de alta letalidad que afecta las vías aéreas superiores del ser humano exclusivamente y que es prevenible mediante la vacunación ${ }^{1}$. Este microorganismo se adhiere fuertemente a las células ciliadas a través de adhesinas. La toxina pertussis y la hemaglutinina filamentosa son las proteínas de ataque más importantes, aunque las proteínas fimbriales pertactina y BrkA (por sus siglas en inglés Bordetella resistance to killing) también participan en este proce$\mathrm{so}^{2,3}$. Las manifestaciones clínicas en lactantes y niños no inmunizados se dividen en tres fases típicas: catarral, paroxística y convalecencia. La letalidad en lactantes es alta: 400 por cada millón de nacidos vivos, mientras que en niños de 1 a 3 años es de 3 a 10 por cada millón de nacidos vivos ${ }^{4}$; en adolescentes y adultos, la gravedad suele ser muy baja ${ }^{5}$.

El mecanismo de transmisión de esta bacteria es mediante aerosoles durante los accesos de tos. Se ha calculado que el $80 \%$ de los contactos cercanos no inmunizados de un caso de tosferina pueden adquirir la infección, por lo que se considera una enfermedad altamente contagiosa ${ }^{2}$.

A pesar de los esfuerzos para prevenir esta enfermedad mediante la vacunación, la tosferina continúa presentando repuntes cada 3 a 4 años, y en años recientes, su incidencia ha ido en aumento ${ }^{3}$. La Organización Mundial de la Salud estima que la incidencia anual de tosferina a nivel mundial es de 20 a 40 millones de casos, y es responsable de 89,000 muertes anuales. Sin embargo, estudios de modelaje estiman alrededor de 24.1 millones de casos y 160 mil muertes en 2014 a escala mundial, con mayor mortalidad en los menores de 1 año de edad 6 .
Los grupos etarios afectados por esta enfermedad han cambiado: antes de la implementación de la vacuna de la difteria, Bordetella pertussis (tosferina) y tétanos (DPT), en la década de los 40 , los más afectados eran preescolares y escolares; sin embargo, actualmente son los adolescentes, adultos y los lactantes menores de 6 meses. En los dos primeros grupos, esto se debe a la pérdida de la inmunidad específica con el paso de los años, mientras que en los lactantes es por contagio a partir de los adultos ${ }^{7}$.

Si bien la gravedad de la tosferina es menor en adolescentes y adultos, estos fungen como reservorio y pueden transmitir la infección a neonatos y lactantes que aún no están protegidos por la vacuna contra $B$. pertussis y tienen un riesgo mayor de sufrir complicaciones o fallecer por esta enfermedad 5 . De hecho, se ha reportado que mueren del 1 al $3 \%$ de los menores de 6 meses que padecen tosferina. La mayoría de muertes por esta enfermedad que se dan en este grupo de edad es en quienes aún no han completado su esquema inicial de vacunación contra $B$. pertussis ${ }^{8-11}$. Entre los factores de riesgo para la mortalidad se encuentran el bajo peso al nacer, menor edad gestacional y menor edad al inicio de los síntomas, así como cuentas más altas de células blancas y linfocitos ${ }^{10}$.

En hospitales pediátricos, especialmente de lugares donde la vacunación contra la tosferina en adultos no es obligatoria, la transmisión puede ser especialmente crítica: los pacientes suelen encontrarse con los factores de riesgo mencionados y están en contacto cercano con adultos permanentemente. Los reportes de brotes de tosferina en hospitales muestran que el caso índice es, con frecuencia, una persona adulta en quien la enfermedad se manifiesta únicamente con tos persistente de semanas a meses de evolución $n^{12}$.

Actualmente, según el esquema nacional de vacunación en México, la prevención en contra de la 
tosferina es por medio de la aplicación de vacunas en niños de 2 meses a 12 años de edad. Las vacunas utilizadas en México son la pentavalente acelular y la vacuna DPT celular ${ }^{13}$. Según el esquema nacional de vacunación, la vacuna pentavalente debe ser aplicada a los 2, 4, 6 y 18 meses de edad, mientras que la DPT celular debe ser aplicada a los 4 y 12 años. Desafortunadamente, no existen lineamientos para la aplicación de refuerzos o revacunación después de los 12 años ${ }^{13}$.

El diagnóstico de la $B$. pertussis en adultos puede ser difícil debido a la baja especificidad de los síntomas en esta población. Un análisis por reacción en cadena de la polimerasa en el momento agudo es el método de diagnóstico ideal (sensibilidad del $61 \%$ y especificidad del $88 \%)^{14}$. Sin embargo, es posible que no se sospeche de tosferina dado el cuadro clínico ambiguo que puede pasar desapercibido ${ }^{14}$. El conocer el perfil serológico en una población determinada puede brindar información, tanto de infección reciente como de inmunidad secundaria a la vacunación o a la infección ${ }^{15}$.

La participación del personal de salud de hospitales pediátricos cobra relevancia en la cadena de transmisión hacia estos grupos vulnerables quienes, además, se encuentran con otros procesos mórbidos subyacentes. En 2015, Cunegundes, et al. reportaron infecciones recientes de $B$. pertussis en médicos y enfermeras de un hospital local de Brasil ${ }^{15}$. De manera relevante, no se encuentra normada ninguna vacuna contra B. pertussis para personal de la salud en México ${ }^{16}$. Dado lo anterior y para conocer la relevancia del problema, se realizó este estudio, con el propósito de conocer la seroprevalencia de $B$. pertussis en el personal de salud de un hospital pediátrico de enseñanza en un país donde la vacuna DPT aún no se considera obligatoria para los empleados sanitarios.

\section{Métodos}

El Hospital Infantil de México Federico Gómez (HIMFG) es un hospital de tercer nivel de atención, de enseñanza, que atiende población pediátrica de escasos recursos y sin seguridad social. De mayo a septiembre de 2015, se invitó a participar a personal de enfermería, médicos y residentes de los servicios de hospitalización, incluyendo la Unidad de Cuidados Intensivos Neonatales (UCIN), Unidad de Terapia Intensiva Pediátrica (UTIP) y Urgencias. Se tomó una proporción de cada turno: alrededor del 30\% fue personal del turno matutino, el $30 \%$, del turno vespertino, el $20 \%$, de velada A y el $20 \%$, de velada B. El personal que se negó a participar fue debido, principalmente, a no aceptar la toma de la muestra. Aproximadamente, tres personas se negaron a la toma y siete no cumplieron con los criterios de selección o su muestra no fue suficiente.

A cada participante se le tomó una muestra de sangre para detección de anticuerpos IgG antitoxina de pertussis (anti-TP). Se interrogó a los participantes y ninguno de los entrevistados refirió haber sido vacunado durante su vida adulta. Los anticuerpos anti-B. pertussis se midieron mediante un ensayo de inmunoabsorción-enzimática ${ }^{15}$. Si los títulos de anticuerpos resultaron superiores a $50 \mathrm{Ul} / \mathrm{ml}$, se consideró como infección reciente; entre 4-49 UI/ml, como exposición a $B$. pertussis en el último año y $<4 \mathrm{UI} / \mathrm{ml}$ negativo. Además, cada participante contestó un cuestionario para evaluar datos sociodemográficos, historia de tos de más de 2 semanas y tiempo que llevaban laborando en el hospital y en el servicio actual.

Se calculó un tamaño de muestra de 85 participantes basándose en el estudio de Deville, et al., donde se detectó el 32\% de positividad para anticuerpos anti-TP con un error alfa de $0.05^{17}$.

El análisis estadístico se realizó en el programa SPSS versión 22. Las variables continuas se analizaron utilizando $U$ de Mann Whitney. Las variables categóricas fueron analizadas con la prueba $\chi^{2}$ de Pearson. Se consideró como estadísticamente significativo un valor de $p<0.05$.

El comité de ética del HIMFG aprobó el protocolo del estudio. Se obtuvo consentimiento informado de todos los participantes incluidos en el proyecto.

\section{Resultados}

Se incluyeron 93 individuos, de los cuales tres no aceptaron participar por temor a la toma de muestra de sangre y siete no cumplieron con los criterios de selección o su muestra no fue suficiente. De los individuos incluidos, el $79.85 \%$ fue personal de enfermería y el resto (14.15\%), médicos residentes, con mediana de edad de 35 años (rango intercuartil (RI): 29-42.5) y predominio del sexo femenino (90.3\%). El tiempo promedio de antigüedad en el hospital fue de 8 años (RI: 3-19 años) y el tiempo en su servicio de adscripción, de 2 años (RI: 1-6 años). El 54.8\% de los participantes pertenecía al turno matutino, el $33.3 \%$, al turno vespertino, el $3.2 \%$, al nocturno y el $8 \%$, al turno mixto. El $21.5 \%$ de los participantes se encontraba laborando en el área de urgencias, el 8.6\%, en la UTIP, el $6.5 \%$, en UCIN y el resto, en otras áreas de 
Tabla 1. Características sociodemográficas de la población

\begin{tabular}{|c|c|}
\hline Variable & $n=93$ \\
\hline Edad (años)* & $35(29-42.5)$ \\
\hline $\begin{array}{l}\text { Sexo } \\
\text { Femenino }\end{array}$ & $\begin{array}{c}n(\%) \\
84(90.3)\end{array}$ \\
\hline $\begin{array}{l}\text { Cargo } \\
\text { Personal de enfermería } \\
\text { Médico residente }\end{array}$ & $\begin{array}{c}n(\%) \\
85(91.4) \\
8(8.6)\end{array}$ \\
\hline Tiempo en el servicio (años)* & $2(1-6)$ \\
\hline Tiempo de ingreso (años) ${ }^{*}$ & $8(3-19)$ \\
\hline $\begin{array}{l}\text { Turno } \\
\text { Matutino } \\
\text { Vespertino } \\
\text { Nocturno } \\
\text { Mixto }\end{array}$ & $\begin{array}{c}n(\%) \\
51(54.8) \\
31(33.3) \\
3(3.2) \\
8(8.6)\end{array}$ \\
\hline $\begin{array}{l}\text { Servicio } \\
\text { Hospitalización } \\
\text { Urgencias } \\
\text { UTIP } \\
\text { UCIN }\end{array}$ & $\begin{array}{c}n(\%) \\
59(63.4) \\
20(21.5) \\
8(8.6) \\
6(6.5)\end{array}$ \\
\hline Tos $>2$ semanas & $24(25.8)$ \\
\hline
\end{tabular}

*Las variables se expresan en mediana con rango intercuartil.

UCIN: Unidad de Cuidados Intensivos Neonatales; UTIP: Unidad de Terapia Intensiva Pediátrica.

hospitalización. El $25.8 \%$ de los participantes reportaron historia de tos de más de dos semanas de duración (Tabla 1).

De toda la muestra, se encontraron títulos detectables de anticuerpos anti-TP en el $18.3 \%(n=17)$ de los participantes con intervalo de confianza al 95\% (IC 95\%) de 11.3-29.4: el 47\% mostró títulos de anticuerpos entre $4-49 \mathrm{UI} / \mathrm{ml}$ y el $53 \%$, cifras mayores de $50 \mathrm{Ul} / \mathrm{ml}$ como marca de infección reciente (Fig. 1) ${ }^{12}$.

Entre los datos basales, la única variable que mostró diferencias estadísticamente significativas entre los seropositivos y seronegativos fue el sexo: una mayor prevalencia de hombres entre los seropositivos para B. pertussis ( $23.6 \%$ vs. $6.6 \% ; p=0.033)$. La edad tuvo una tendencia a ser menor en los seropositivos $(p=0.066)$. Aunque la frecuencia de trabajadores del turno matutino y vespertino, así como de personal de enfermería y de las áreas de UTIP y UCIN fue mayor entre los seronegativos para $B$. pertussis, la diferencia no resultó estadísticamente significativa $(p \geq 0.1)$. La antigüedad de los trabajadores en sus áreas, así como en el hospital, fue mayor entre los sujetos seronegativos. Sin embargo, la diferencia tampoco resultó estadísticamente significativa (Tabla 2).

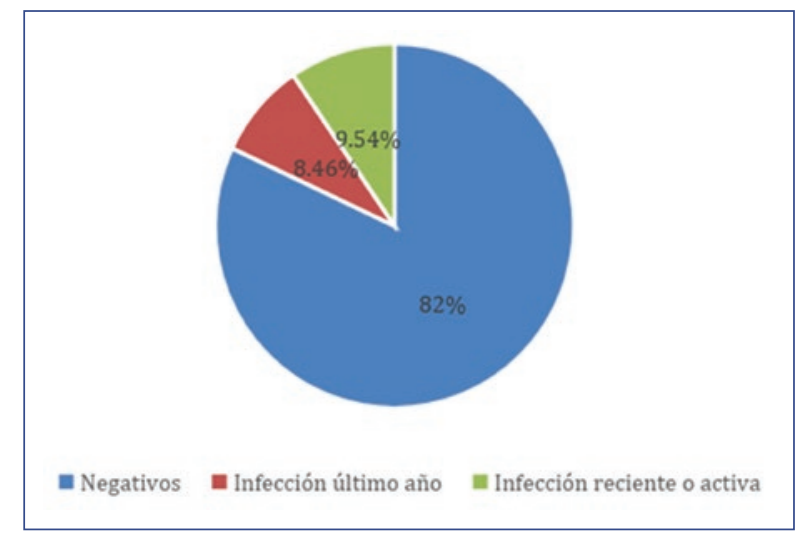

Figura 1. Perfil serológico para Bordetella pertussis en médicos y personal de enfermería del Hospital Infantil de México Federico Gómez. Se consideró como positivo para $B$. pertussis cuando los títulos de anticuerpos anti-TP (toxina pertussis) fueron $\geq 4 \mathrm{Ul} / \mathrm{ml}$ y negativo cuando los anticuerpos anti-TP fueron $<4 \mathrm{UI} / \mathrm{ml}$.

Los anti-TP entre 4-49 UI/ml corresponden a sujetos expuestos a $B$. pertussis en el último año antes del estudio. Títulos mayores a $50 \mathrm{UI} / \mathrm{ml}$ corresponden a los sujetos con una infección reciente 0 activa.

\section{Discusión}

En este estudio en el que participaron médicos residentes y personal de enfermería que laboran en un hospital pediátrico de referencia, se encontró que el $82 \%$ no contaba con inmunidad alguna contra la tosferina. Interesantemente, cerca de uno de cada diez mostraron serología compatible con una infección reciente, y el personal del Servicio de Urgencias fue el más afectado.

Aunque la vacunación contra $B$. pertussis forma parte del esquema nacional de vacunación, la duración de la inmunidad es menor a una década. La última dosis se aplica a los 4 años de edad, por lo que los adolescentes y adultos vuelven a ser susceptibles a sufrir la infección, como se ha demostrado en este estudio ${ }^{13,14}$.

Asimismo, el 9.7\% de los trabajadores evaluados mostraron un título de anti-TP de más de $50 \mathrm{Ul} / \mathrm{ml}$, compatible con una infección reciente, lo cual indica que existe una exposición frecuente a este agente. En 2015, Cunegundes, et al. reportaron una seroprevalencia de $6.4 \%$ de títulos altos de anticuerpos, prueba de una infección reciente, en médicos y enfermeras ${ }^{15}$. El hecho de encontrar personal de salud con serología compatible con infección es de suma relevancia, porque se ha encontrado que en brotes de B. pertussis en niños, en muchas ocasiones, el caso índice es un adulto del personal de salud ${ }^{14-16}$.

Otros estudios han mostrado una frecuencia aún mayor de infección por B. pertussis en el personal de salud. 
Tabla 2. Comparación de los sujetos con anticuerpos positivos y negativos

\begin{tabular}{|c|c|c|c|}
\hline \multirow[t]{2}{*}{ Variable } & Negativos & Positivos & \multirow[t]{2}{*}{$\mathbf{p}^{*}$} \\
\hline & $(n=76)$ & $(n=17)$ & \\
\hline Edad en años, mediana (RI) & $36.5(30-44)$ & $32(29.5-36.5)$ & 0.066 \\
\hline Sexo femenino, $\mathrm{n}(\%)$ & $71(93.4 \%)$ & $13(76.4 \%)$ & 0.033 \\
\hline $\begin{array}{l}\text { Cargo, n (\%) } \\
\text { Personal de enfermería } \\
\text { Residentes } \\
\text { Tiempo en el servicio, mediana (RI) } \\
\text { Tiempo de ingreso, mediana (RI) }\end{array}$ & $\begin{array}{c}70(92.1 \%) \\
6(7.8 \%) \\
2.5(1-7) \\
8.5(3-21)\end{array}$ & $\begin{array}{c}15(88.2 \%) \\
2(11.8 \%) \\
2(1-4.5) \\
7(2.5-11)\end{array}$ & $\begin{array}{l}0.632 \\
0.239 \\
0.182\end{array}$ \\
\hline $\begin{array}{l}\text { Turno, } n(\%) \\
\text { Matutino } \\
\text { Vespertino } \\
\text { Nocturno } \\
\text { Mixto }\end{array}$ & $\begin{array}{c}42(55.2 \%) \\
26(34.2 \%) \\
2(2.6 \%) \\
6(7.8 \%)\end{array}$ & $\begin{array}{c}9(52.9 \%) \\
5(29.4 \%) \\
1(5.8 \%) \\
2(11.7 \%)\end{array}$ & 0.722 \\
\hline $\begin{array}{l}\text { Servicio, n (\%) } \\
\text { Hospitalización } \\
\text { Urgencias } \\
\text { UTIP } \\
\text { UCIN }\end{array}$ & $\begin{array}{c}48(63.1 \%) \\
15(19.7 \%) \\
7(9.2 \%) \\
6(7.8 \%)\end{array}$ & $\begin{array}{c}11(64.7 \%) \\
5(29.4 \%) \\
1(5.8 \%) \\
0\end{array}$ & 0.664 \\
\hline Historia de tos $>2$ semanas, $\mathrm{N}(\%)$ & $20(26.3 \%)$ & $4(23.5 \%)$ & 0.54 \\
\hline
\end{tabular}

${ }^{*} p$ significativa cuando los valores son $<0.05$.

Se consideró como positivo para B. pertussis cuando los títulos de anticuerpos anti-TP (toxina pertussis) fueron $>4 \mathrm{UI} / \mathrm{ml}$ y negativo cuando fueron $<4 \mathrm{UI} / \mathrm{ml}$. Las variables se expresan en mediana, rango intercuartil y porcentaje. Para la comparación se utilizó U de Mann Whitney y $\chi^{2}$.

UCIN: Unidad de Cuidados Intensivos Neonatales; UTIP: Unidad de Terapia Intensiva Pediátrica.

Deville, et al. realizaron determinaciones seriadas de anticuerpos contra este agente en 51 trabajadores de la salud durante 5 años consecutivos, y encontraron una tasa anual de infección de entre $24-43 \%$, determinada por un aumento significativo en el título de anticuerpos ${ }^{17}$.

Un estudio metacéntrico llevado a cabo por Rodríguez, et al. en España mostró una seroprevalencia del $31.2 \%$ en el personal de la salud: el $3.3 \%$ de los participantes tenía títulos de anticuerpos que demostraban datos de infección reciente ${ }^{18}$.

Los reportes sobre el comportamiento epidemiológico de la tosferina en México son escasos. Además del estudio llevado a cabo en escuelas secundarias en la Ciudad de México ${ }^{10}$, existe una encuesta seroepidemiológica llevada a cabo en 2010 en 3,344 individuos de diversas áreas del país, que reveló una seropositividad del $47.4 \%$, mayor en niños (59.3\%) en comparación con adolescentes y adultos. Por otro lado, el $21.8 \%$ de la población analizada mostraba títulos de anticuerpos como prueba de infección o vacunación reciente ${ }^{16}$.

Es posible que las diferencias de seroprevalencia entre el presente estudio y los reportes anteriores sean secundarias a las fluctuaciones propias de la enfermedad, que presenta repuntes cíclicos cada 3 a 4 años, o a la diferencia en la prevalencia entre diferentes países, o incluso, en el apego a las medidas de prevención de infecciones respiratorias ${ }^{5}$. En el periodo de estudio, la adherencia a la higiene de manos en el hospital fue del 61.6\% (IC 95\%: 60-65). Sin embargo, no se cuenta con tal información en las demás investigaciones que han abordado el problema.

El hecho de que el Servicio de Urgencias haya sido el servicio con una mayor proporción de participantes con anticuerpos detectables, coincide con lo encontrado por otros autores ${ }^{16}$. El Servicio de Urgencias es el primer contacto que se tiene con el paciente. Dado que el cuadro clínico de tosferina en muchos casos es inespecífico, con frecuencia no se sospecha el diagnóstico de manera inicial, lo cual, aunado a la falta de apego a medidas de precaución, como higiene de manos y uso de cubre bocas, facilitaría la transmisión al personal de salud.

La historia de tos de más de 2 semanas de evolución, con una prevalencia del $23.5 \%$ en los participantes con anticuerpos anti-TP detectables, no fue significativamente asociada con la seropositividad por B. pertussis. Como se describió previamente, el cuadro clínico de tosferina en los adultos es con frecuencia inespecífico, lo que dificulta el diagnóstico y manejo oportunos en esta población, en la que más de tres cuartas partes de los 
casos no presentaban manifestaciones clínicas, y propician la transmisión en el hospital ${ }^{13,19}$.

Dada la baja prevalencia de seropositividad documentada en este estudio, que refleja una gran proporción de población susceptible entre los trabajadores de salud del hospital, así como la dificultad para el diagnóstico clínico temprano ${ }^{17,20}$, los resultados obtenidos sugieren que es necesario implementar estrategias para la prevención de la tosferina. Entre estas estrategias se encuentran los refuerzos de vacunación en el personal de salud $^{21}$, como se recomienda en países como Alemania y EE.UU.22. Esto permitirá proteger tanto a los trabajadores de salud como a los pacientes, y evitará brotes hospitalarios que pueden causar morbimortalidad sustancial, especialmente en los lactantes menores.

Como conclusiones, el personal de salud muestreado mostró en su mayoría ausencia de anticuerpos protectores contra $B$. pertussis, además de que en cerca del $10 \%$ se encontraron títulos de anticuerpos de infección reciente sin antecedentes de datos clínicos compatibles. Ambos hallazgos sugieren que el personal de salud está en riesgo de contraer la enfermedad y, especialmente, de transmitirla a los lactantes atendidos en el hospital, quienes pueden sufrir complicaciones graves, o incluso fallecer por esta causa. Este estudio cobra relevancia dado que brinda la pauta para sugerir que las políticas actuales de vacunación se modifiquen para determinar la obligatoriedad de la vacunación y reforzar las medidas de prevención de transmisión de B. pertussis en el personal de salud que atiende población pediátrica, con el fin de evitar transmisión de la enfermedad, especialmente a poblaciones vulnerables.

\section{Responsabilidades éticas}

Protección de personas y animales. Los autores declaran que para esta investigación no se han realizado experimentos en seres humanos ni en animales.

Confidencialidad de los datos. Los autores declaran que han seguido los protocolos de su centro de trabajo sobre la publicación de datos de pacientes.

Derecho a la privacidad y consentimiento informado. Los autores han obtenido el consentimiento informado de los pacientes o individuos referidos en el artículo. Este documento obra en poder del autor de correspondencia.

\section{Conflicto de intereses}

Los autores declaran no tener ningún conflicto de intereses.

\section{Financiamiento}

Hospital infantil de México Federico Gómez.

\section{Bibliografía}

1. Beltrán Silva S, Cervantes Apolinar Y, Cherry JD, Geffen D, Conde González C, Gentile A, et al. Consenso para el diagnóstico clínico y microbiológico y la prevención de la infección por Bordetella pertussis. Salud Publica Mex. 2011;53:57-65.

2. Alexander EM, Travis S, Booms C, Kaiser A, Fry NK, Harrison TG, et al. Pertussis outbreak on a neonatal unit: identification of a healthcare worker as the likely source. J Hosp Infect. 2008;69:131-4.

3. Centers for Disease Control and Prevention. Pertussis home page. Outbreaks. Disponible en: http://www.cdc.gov/pertussis/outbreaks. $\mathrm{html}$.

4. World Health Organization. Background paper. SAGE April 2014; 2014:82. Disponible en: http://www.who.int/immunization/sage/meetings/2014/april/1_Pertussis_background_FINAL4_web.pdf?ua=\%0Ahttp://www.who.int/immunization/sage/meetings/2014/april/1_Pertussis_ background_FINAL4_web.pdf.

5. World Health Organization. Pertussis vaccines: WHO position paperSeptember 2015. Wkly Epidemiol Rec. 2015;90(35):433-58.

6. Yeung KHT, Duclos P, Nelson EAS, Hutubessy RCW. An update of the global burden of pertussis in children younger than 5 years: a modelling study. Lancet Infect Dis. 2017;17:974-80.

7. Hewlett E, Edwards K. Pertussis-not just for kids. N Engl J Med. 2005;352:1215-22.

8. Winter K, Zipprich J, Harriman K, Murray EL, Gornbein J, Hammer SJ, et al. Risk factors associated with infant deaths from pertussis: a case-control study. Clin Infect Dis. 2015;61:1099-106.

9. Maltezou HC, Ftika L, Theodoridou M. Nosocomial pertussis in neonatal units. J Hosp Infect. 2013;85:243-8.

10. Follmann D, Qin J, Guerrero ML, Breugelmans JG, Rosales Pedraza G, Gessner BD, et al. Estimating the burden of pertussis in Mexican adolescents from paired serological data by using a bivariate mixture model. J R Stat Soc Ser C Appl Stat. 2014;63:621-37.

11. Wirsing Von König $\mathrm{CH}$, Halperin S, Riffelmann M, Guiso N. Pertussis of adults and infants. Lancet Infect Dis. 2002;2:744-50.

12. Wendelboe AM, Van Rie A, Salmaso S, Englund JA. Duration of immunity against pertussis after natural infection or vaccination. Pediatr Infect Dis J. 2005;24:58-61.

13. Centro Nacional para la Salud de la Infancia y Adolescencia. México: Programa Sectorial de Salud; 2018. Programa de acción especifico vacunación universal. Disponible en: http://www.censia.salud.gob.mx/ contenidos/descargas/transparencia/especiales/PAE_Vacunacion_Universal_PAE_final_final.pdf.

14. Zouari A, Smaoui $H$, Kechrid A. The diagnosis of pertussis: which method to choose? Crit Rev Microbiol. 2012;38:111-21.

15. Cunegundes KSA, de Moraes-Pinto MI, Takahashi TN, Kuramoto DAB, Weckx LY. Bordetella pertussis infection in paediatric healthcare workers. J Hosp Infect. 2015;90:163-6.

16. Conde-Glez C, Lazcano-Ponce E, Rojas R, DeAntonio R, Romano-Mazzotti L, Cervantes Y, et al. Seroprevalence of Bordetella pertussis in the Mexican population: a cross-sectional study. Epidemiol Infect. 2014; 142:706-13.

17. Deville JG, Cherry JD, Christenson PD, Pineda E, Leach CT, Kuhls TL, et al. Frequency of unrecognized Bordetella pertussis infections in adults. Clin Infect Di s. 1995;21:639-42.

18. Rodríguez de la Pinta ML, Castro Lareo MI, Ramon Torrell JM, García de Lomas J, Devadiga R, Reyes J, et al. Seroprevalence of pertussis amongst healthcare professionals in Spain. Vaccine. 2016;34:1109-14.

19. De Serres G, Shadmani R, Duval B, Boulianne N, Dery P, Fradet MD, et al. Morbidity of pertussis in adolescents and adults. J Infect Dis. 2000;182:174-9.

20. Sandora TJ, Gidengil CA, Lee GM. Pertussis vaccination for health care workers. Clin Microbiol Rev. 2008;21:426-34.

21. Bechini A, Tiscione E, Boccalini S, Levi M, Bonanni P. Acellular pertussis vaccine use in risk groups (adolescents, pregnant women, newborns and health care workers): a review of evidences and recommendations. Vaccine. 2012;30:5179-90.

22. Esolen LM, Kilheeney KL. A mandatory campaign to vaccinate health care workers against pertussis. Am J Infect Control. 2013;41:740-2. 